\title{
More Live Births in Primary Subfertile Intracytoplasmic Sperm Injection-Treated Women with High Normal TSH Levels
}

\author{
Constance Repelaer van Driel-Delprat ${ }^{a} \quad$ Eveline van Dam ${ }^{b}$ Peter van de Ven ${ }^{c}$ \\ Khadija Aissa $^{a} \quad$ Melanie ter Haar ${ }^{a}$ Yikke Feenstra $^{a} \quad$ Aletta de Roos $^{a}$ \\ Gaby Beelen $^{\mathrm{a}}$ Roel Schats ${ }^{\mathrm{a}}$ Cornelis Lambalk ${ }^{\mathrm{a}}$ \\ aDepartment of Obstetrics, Gynaecology and Reproductive Medicine, Division of Reproductive Medicine, \\ Amsterdam Reproduction \& Development, Amsterdam UMC, Vrije Universiteit Amsterdam, Amsterdam, \\ The Netherlands; ${ }^{b}$ Department of Internal Medicine, Division of Endocrinology, Amsterdam UMC, Vrije Universiteit \\ Amsterdam, Amsterdam, The Netherlands; ' Department of Epidemiology and Biostatistics, Amsterdam UMC, \\ Vrije Universiteit Amsterdam, Amsterdam, The Netherlands
}

\section{Keywords}

Reproductive outcome - Thyroid-stimulating hormone · Intracytoplasmic sperm injection · Primary subfertility

\begin{abstract}
Objectives: The aim of this study was to analyze the fertility outcome in intracytoplasmic sperm injection (ICSI)-treated women across normal range thyroid-stimulating hormone (TSH) levels. Published results are inconclusive about optimal TSH levels and fertility. Design: This is a retrospective cohort study in 752 ICSI-treated women with predominantly severe male factor subfertility, starting treatment between the first of January 2008 and the first of March 2012 with a follow-up until 2014. Participants/Materials, Setting, Methods: Women aged 22-45 years with TSH 0.3-4.5 mlU/L without thyroid hormone substitution were included in Amsterdam UMC, Vrije Universiteit, Amsterdam, The Netherlands, an iodine-sufficient area. Demographic and baseline characteristics were compared between groups of patients based on TSH, using one-way ANOVA, Kruskal-Wallis ANOVA, and $\mathrm{X}^{2}$ test. The patient was the unit of analysis: all cumulative cycles per patient were analyzed up to and including the first ongoing pregnancy. The primary outcome was a cumulative
\end{abstract}

live birth rate. Clinical pregnancy rate, pregnancy loss, and ongoing pregnancy rate were secondary outcomes. The $x^{2}$ test and logistic regression were used to compare interquartile groups while adjusting for confounders. Logistic regression was used with the natural logarithm of TSH as a continuous predictor. Primary and secondary subfertile women were analyzed separately. Results: Analysis of the total cohort $(n=752)$ showed no difference in fertility outcomes across the normal TSH range. The cumulative live birth rate for the 4 groups of primary subfertile women $(n=455)$ was $76 \%$ in the upper TSH quartile compared to $56 \%, 60 \%$, and $59 \%$ in the lower TSH quartiles. Limitations: Levels of thyroxine and presence of thyroid autoimmunity were not measured in this retrospective cohort study. Conclusions: The observation that a higher live birth rate was found in primary subfertile ICSI-treated women with high but allegedly normal TSH levels contributes to the hypothesis that in certain subfertile women in addition to a male factor, female factors such as subtle hypothyroidism and/or thyroid autoimmunity may play a role in keeping them from conception, which can be overcome by the process of ICSI.

(c) 2021 The Author(s).

Published by S. Karger AG, Basel
(C) 2021 The Author(s)

Published by S. Karger AG, Basel

This is an Open Access article licensed under the Creative Common Attribution-NonCommercial-4.0 International License (CC BY-NC) (http://www.karger.com/Services/OpenAccessLicense), applicable to the online version of the article only. Usage and distribution for commercial purposes requires written permission.
Correspondence to:

Constance Repelaer van Driel-Delprat, c.delprat@amsterdamumc.nl 


\section{Introduction}

Subclinical hypothyroidism, defined as a thyroidstimulating hormone (TSH) level above the reference range with thyroxine (FT4) within the reference range, affects $2-4 \%$ of women of fertile age. Since the beginning of this century, the upper limit of the reference range for the definition of subclinical hypothyroidism in subfertile women is a topic of debate. TSH levels between $2.5 \mathrm{mIU} / \mathrm{L}$ and $4.5 \mathrm{mIU} / \mathrm{L}$ are associated with diminished capability to ovulate, fertilize, or nidate [1] and might reflect an inability to fulfill the up to $50 \%$ increase in production of thyroid hormones required for an uncomplicated ongoing pregnancy [2], especially in the presence of thyroid autoimmunity. The prevalence of these high normal TSH levels in assisted reproductive techniques (ART)-treated women is 4 times higher $(20-26 \%)[3,4]$ than those in the general population (5\%) [5]. The prevalence of thyroid autoimmunity is higher in subfertile women with TSH above $2.5 \mathrm{mIU} / \mathrm{L}$ : the probability of having thyroid peroxidase antibodies (TPO-Abs) doubles from slightly under 0.2 with TSH $2.5 \mathrm{mIU} / \mathrm{L}$ to slightly above 0.4 with TSH of $4.5 \mathrm{mIU} / \mathrm{L}$ [6]. About $8 \%$ of women with recurrent miscarriage or subfertility and thyroid autoimmunity developed thyroid dysfunction during pregnancy [6, 7]. The area of dialog, by lack of evidence, is reflected in recommendations of both the American Thyroid Association [8] and the American Society for Reproductive Medicine [9], pointing at the possible benefit of levothyroxine supplementation for women undergoing IVF or ICSI with TSH $>2.5 \mathrm{mIU} / \mathrm{L}$ with or without thyroid autoimmunity, however not recommending screening or treatment with levothyroxine [8].

So far, predominantly retrospective observational studies comparing ART-treated women with high normal TSH levels (2.5-4.5 mIU/L) to women with low normal TSH levels (0.3-2.49 mIU/L) report a lower fertilization rate [10] and impaired embryo quality with increased TSH or presence of thyroid autoantibodies $[11,12]$ and conflicting results concerning pregnancy rate, pregnancy loss, and live birth rate [13-15]. The hypothesized pathophysiological mechanism involved is shifting from thyroid dysfunction as reflected in TSH levels to thyroid dysfunction related to (thyroid) autoimmunity. Published studies are heterogeneous and based on TSH levels alone or combined with the presence of thyroid autoantibodies [16]. Both women with high normal TSH values prior to ART and women with TPO-Abs are shown to have an increased rate of pregnancy loss and unsuccessful pregnancy after ART [17]. However, a recent review [13] and
2 randomized controlled trials for supplementation of levothyroxine in euthyroid women with TPO-Abs showed no decline in pregnancy loss $[7,13,18]$. Most of these studies were in a mixed cohort of IVF and ICSI patients. Often both IVF and ICSI [18] or ICSI treatments only [19, 20] are involved. With the possible impact of thyroid hormones or autoantibodies on the process of fertilization and/or early embryo development [20,21], it is of importance to analyze these 2 treatment options separately. Primary and secondary subfertile women were so far not analyzed separately, although secondary subfertility is associated with an increased prevalence of thyroid autoimmunity, disappearing after correcting for age [19], and in fecund women, no association of high normal TSH levels and subfertility is observed [22].

Therefore, inconsistent results may be explained partly by a variable TSH upper reference level limit, by analyzing TPO-Ab-positive and TPO-Ab-negative women together, by the variety in ART and its indications, and by analyzing primary and secondary subfertile women together. A potential effect of TSH levels and thyroid autoimmunity on ART outcomes for subgroups of subfertile women might have gone unnoticed in this heterogeneity. In order to explore for whom subclinical thyroid dysfunction might contribute to subfertility, the aim of this study was to analyze fertility outcome in ICSI-treated subfertile women with TSH within the normal reference range, whose subfertility is assigned predominantly to severe male factor.

\section{Patients and Methods}

Study Population and Participants

All subfertile women who started ICSI in Amsterdam UMC, Vrije Universiteit Amsterdam, Netherlands between the first of January 2008 and the first of March 2012 with a follow-up until 2014 were retrospectively reviewed. Data were obtained from paper, electronic patient files, and, in case of ongoing pregnancy, via a routine patient questionnaire.

Inclusion Criteria

Women between 18 and 43 years old, TSH value measured preceding the first stimulation and embryo transfer, with an indication for ICSI: severe male factor $\left(<2 \times 10^{6}\right.$ spermatozoa $)$, or total fertilization failure or fertilization percentage of $<15 \%$ in the previous IVF treatment [23].

\section{Exclusion Criteria}

Use of third party gametes/surrogacy; TSH out of reference range $(<0.3 \mathrm{mIU} / \mathrm{L}$ and $>4.5 \mathrm{mIU} / \mathrm{L})$; a history of thyroid dysfunction; or thyroid hormone substitution. For patient characteristics, TSH values as well as data on BMI and on tobacco and alcohol use nearest to and preceding the first cycle of ICSI treatment were used for analysis. 
A third-generation TSH assay (ECLIA Roche ${ }^{\circledR}$ Cobas 8000) with a reference range of $0.3-4.5 \mathrm{mIU} / \mathrm{L}$ was used. Analysis of primary and secondary subfertile women was performed because of presumed differences [22].

\section{Laboratory and Pregnancy Protocol}

Patients underwent the laboratory IVF/ICSI protocol as outlined by Vergouw et al. [24]. The number of cycles includes fresh and frozen embryo transfers. The number of oocytes, number of 2PN embryos, and fertilization rate include fresh cycles only. Predominantly, 1 embryo was transferred (84\%). In case of double embryo-transfer, the best embryo was used in the analysis. No assisted hatching was performed. Biochemical pregnancy was defined as any rise in hCG 14-16 days after ovulation. Clinical pregnancy was defined as an intrauterine gestation at 6-8 weeks gestation. Ongoing pregnancy was defined as an intrauterine gestation with beating heart, at 11 weeks amenorrhea. Pregnancy loss was defined as a nonviable clinical pregnancy. The live birth rate was defined as a live born child after 24 weeks amenorrhea.

\section{Statistical Analysis}

SPSS 26 was used for statistical analysis. Demographic and baseline characteristics were compared between groups using one-way ANOVA, Kruskal-Wallis ANOVA, and the $\chi^{2}$ test. Pregnancy outcomes are shown with patient as unit of analysis, that is, as cumulative data until ongoing pregnancy per patient and with first cycle per patient. Biochemical, clinical and ongoing pregnancy, live birth rate, and pregnancy loss were determined for patients' first cycle as well as in cumulative data for all patients' cycles of ICSI up until the first ongoing pregnancy. Pregnancy loss is represented as a proportion of clinical pregnancy.

Differences in pregnancy outcomes were tested using logistic regression analysis; patients were categorized into 4 quartiles, each covering $25 \%$ based on TSH values, for uncovering possible ushaped associations of TSH and pregnancy outcomes, such as those described between thyroid hormone levels and IQ levels of the child [25]. In the analyses, the upper quartile group was used as reference. In primary and secondary subfertile women, specific interquartile cutoff values were determined. A second analysis was performed with the natural logarithm of TSH. The first model contained only a linear term. In a second analysis, a quadratic term was added to account for a nonlinear relationship. Analyses were adjusted for age, BMI $\left(<20.9 / 21.0-28.9 / 29-34.9 />35 \mathrm{~kg} / \mathrm{m}^{2}\right)$ [26], use of alcohol (no alcohol [0 units a week]/moderate [1-8 units a week]/heavy [ $>8$ units a week] [27] and tobacco [yes/no]) [28], presence of diminished ovarian reserve $(\mathrm{FSH}>10 \mathrm{IU} / \mathrm{L}$ with an ovulating cycle yes/no) [29], and primary or secondary subfertility defined as having had a previous clinical pregnancy. Odds ratios (ORs) were reported as effect size together with $95 \%$ confidence interval and $p$ values.

\section{Results}

\section{Patient Characteristics}

Nine hundred and three ICSI-treated non-third party women were identified. In 68 of them, no TSH

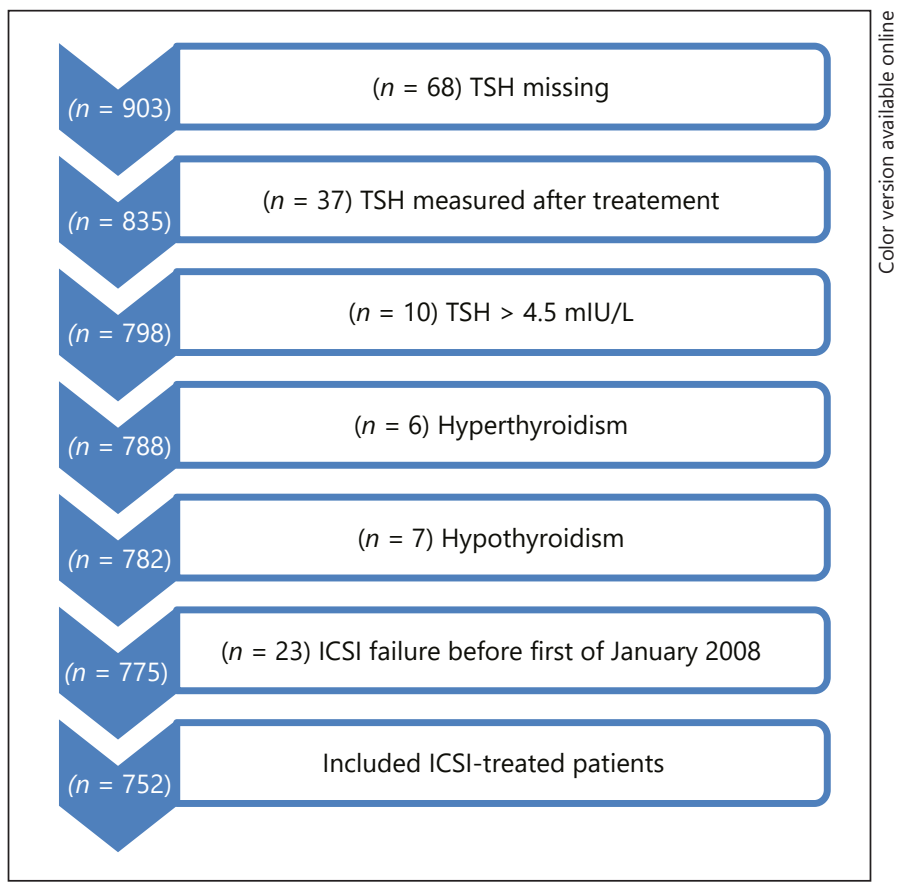

Fig. 1. Flow chart of in- and exclusion criteria of ICSI-treated women. ICSI, intracytoplasmic sperm injection; TSH, thyroidstimulating hormone.

was measured, and in 37, it was measured after treatment. Twenty-three women had thyroid dysfunction, and 23 women had started ICSI treatment for this aimed pregnancy before the inclusion date. 752 women met the inclusion criteria (shown in Fig. 1). Twentythree of them had a previous IVF treatment for unexplained or mild male factor subfertility, with total fertilization failure and 3 with a fertilization rate $<15 \%$. A total of 1,804 cycles until ongoing pregnancy were analyzed. TSH was skewed to the right with a mean of 1.9 $\mathrm{mIU} / \mathrm{L}$. TSH interquartile groups were I: $0.3-1.33$ mIU/L; II: $1.34-1.82 \mathrm{mIU} / \mathrm{L}$; III: $1.83-2.43 \mathrm{mIU} / \mathrm{L}$; and IV: $2.44-4.5 \mathrm{mIU} / \mathrm{L}$. Table 1 shows the distribution of basic characteristics in the interquartile groups, including ART outcomes for all ICSI-treated women. 455 (61\%) women were primary subfertile. In the upper TSH group, the proportion of primary subfertile women was higher than that in the 3 lower TSH groups, albeit not significant. BMI tended to increase with increasing TSH $(p=0.07)$.

Tables 2 and 3 show baseline characteristics of primary and secondary subfertile ICSI-treated patients separately across TSH. Except for a longer duration of hormonal stimulation treatment with both primary and sec- 
Table 1. Patient characteristics of all ICSI-treated women $(n=752)$

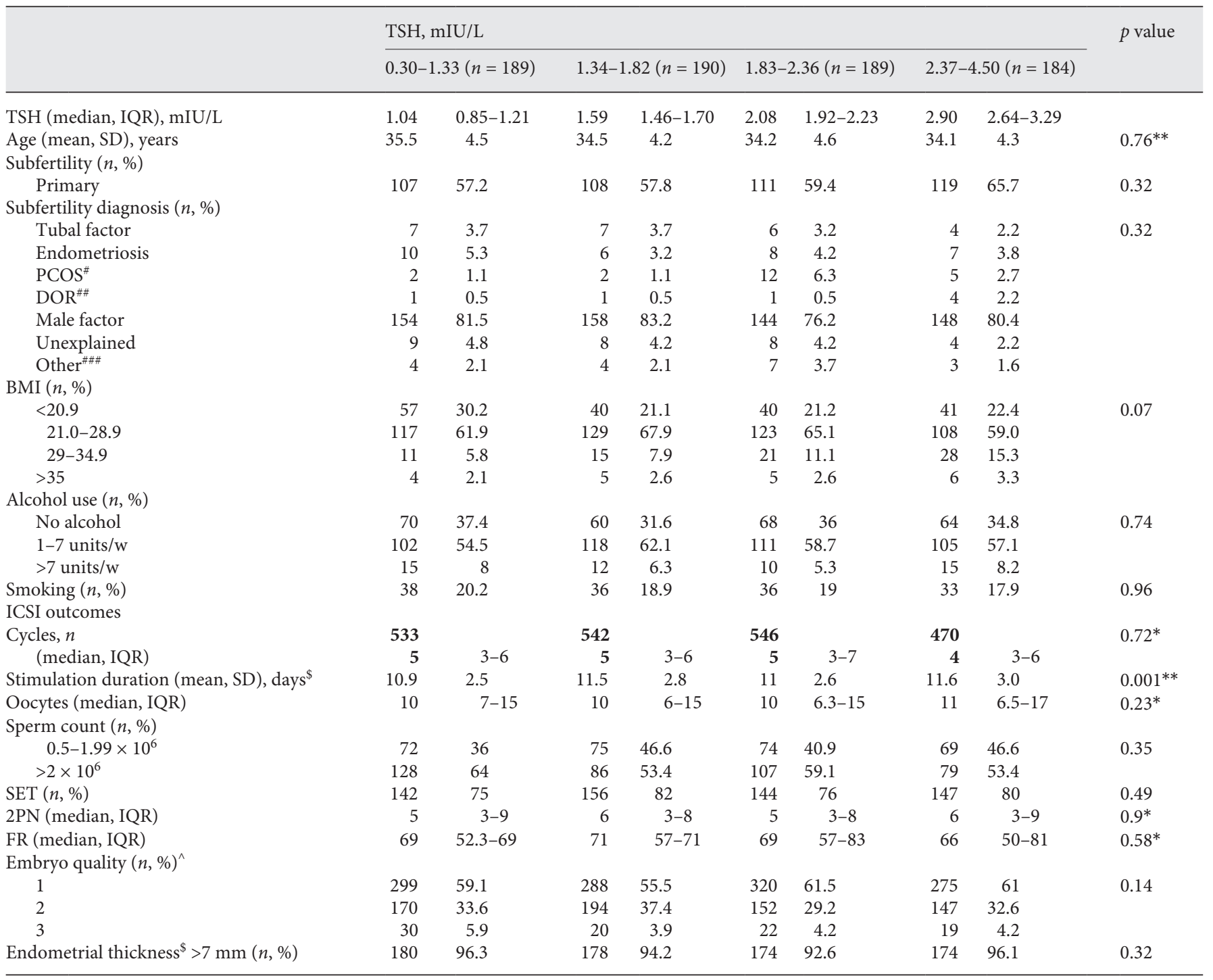

TSH, thyroid-stimulating hormone; IQR, interquartile range; ICSI, intracytoplasmic sperm injection. * Kruskal-Wallis. ** ANOVA. * PCOS: polycystic ovary syndrome. ${ }^{\# \#}$ DOR: diminished ovarian reserve, defined as ovulatory cycles with FSH $>10$ U/L. ${ }^{\# \# \# ~ O t h e r: ~ c o n g e n i t a l ~ u t e r u s ~ a n o m a l y ~ a n d ~ A s h e r m a n ' s ~ s y n d r o m e . ~}$ ${ }^{\wedge}$ Embryo quality: 1, good; 2 , medium; 3, poor. ${ }^{\$}$ First cycle per person.

ondary subfertility, no significant differences could be observed.

\section{Pregnancy Results}

Tables 4-6 show the first cycle and cumulative pregnancy outcomes of all and primary and secondary subfertile ICSI patients separately. No significant differences were seen across the overall group. In the analysis of the primary subfertile women (Table $5 ; n=455$ ), higher ORs for live birth $(p=0.01)$ and the clinical $(p=0.049)$ and ongoing pregnancy rate $(p=0.03)$ were seen in the upper quartile TSH levels, also coming to significance for live birth rate when only the first cycles were analyzed ( $p=$ 0.03 ). Analysis of the natural logarithm of TSH as a continuous predictor showed an increased OR of 1.7 for live birth rate for each point increase of TSH in primary subfertile women $(p=0.03$ ) (Online suppl. Table 1; see www. karger.com/doi/10.1159/000518083 for all online suppl. material). 
Table 2. Patient characteristics of primary subfertile ICSI-treated women $(n=455)$

\begin{tabular}{|c|c|c|c|c|c|c|c|c|c|}
\hline TSH (median, IQR), mIU/L & \multicolumn{8}{|c|}{$\mathrm{TSH}, \mathrm{mIU} / \mathrm{L}$} & $p$ value \\
\hline Age (mean, SD), years & 33.5 & 4.9 & 33.8 & 4.3 & 33.3 & 4.6 & 33.3 & 4.4 & $0.84^{*}$ \\
\hline \multicolumn{10}{|l|}{ ICSI outcomes } \\
\hline Cycles, $n$ & 334 & & 325 & & 324 & & 280 & & $0.50^{*}$ \\
\hline \multicolumn{10}{|l|}{ Embryo quality $(n, \%)^{\wedge}$} \\
\hline 1 & 182 & 56 & 175 & 54.2 & 182 & 58.3 & 175 & 63.9 & 0.21 \\
\hline 2 & 111 & 34.2 & 118 & 36.5 & 96 & 30.8 & 81 & 29.6 & \\
\hline 3 & 22 & 6.8 & 13 & 4.0 & 16 & 5.1 & 11 & 4.0 & \\
\hline $\operatorname{SET}(n, \%)^{\$}$ & 97 & 83 & 99 & 88 & 86 & 76 & 96 & 85 & 0.23 \\
\hline
\end{tabular}

TSH, thyroid-stimulating hormone; IQR, interquartile range; ICSI, intracytoplasmic sperm injection. * Kruskal-Wallis. ** ANOVA. ^ Embryo quality: 1 , good; 2, medium; 3, poor. ${ }^{\$}$ First cycle per person.

Table 3. Patient characteristics of secondary subfertile ICSI-treated women $(n=297)$

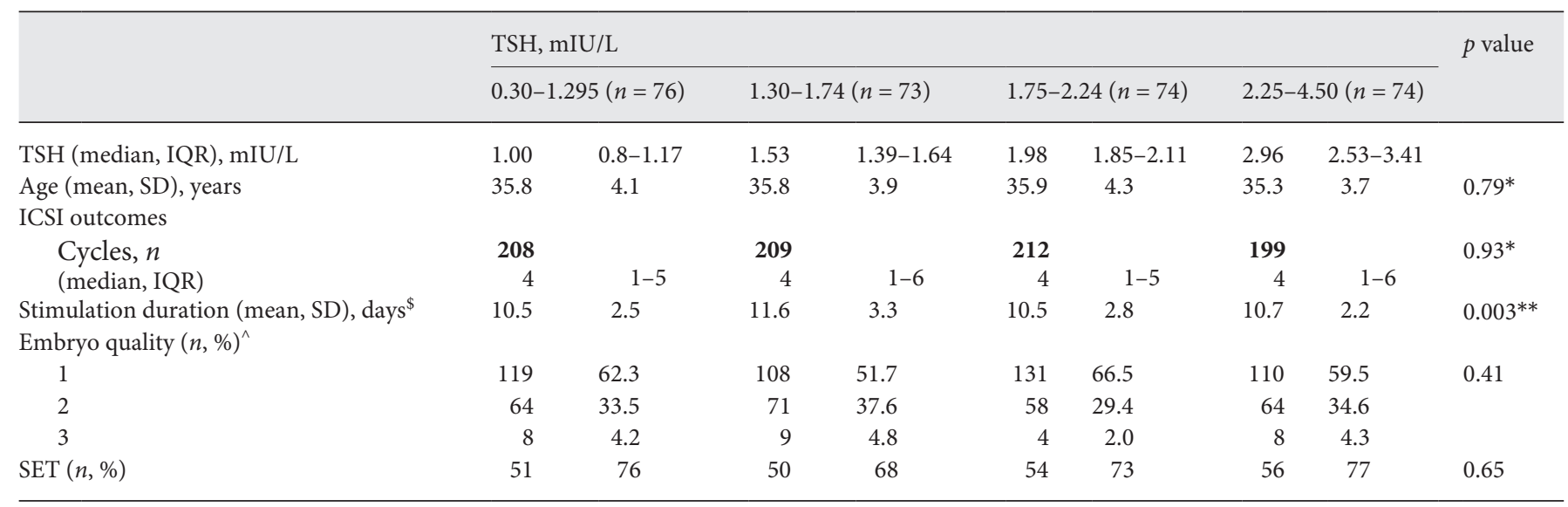

TSH, thyroid-stimulating hormone; IQR, interquartile range; ICSI, intracytoplasmic sperm injection. * Kruskal-Wallis. ** ANOVA. ^ Embryo quality: 1, good; 2, medium; 3, poor. ${ }^{\$}$ First cycle per person.

\section{Discussion}

These retrospective data show higher odds for the live birth rate in a subgroup of primary subfertile women undergoing ICSI with higher TSH within the normal reference range. In our clinic, ICSI is performed because of severe male factor or after a previous IVF treatment with total fertilization failure or a very low fertilization rate $<15 \%$. In these primary subfertile women, high normal TSH might reflect subtle diminished thyroid reserve whether or not related to thyroid autoimmunity [30], which seemingly can be overcome by ICSI. This benefit of different fertility outcomes across TSH ranges is not seen in secondary subfertile women, agreeing with the study of Plowden et al. [22]. This can have its origin in the fact that secondary subfertile women have had the thyroid function tested and/or treated during or after their previous pregnancy.

Autoimmunity comes to expression with increasing age, often from the third decade onwards. Thyroid autoimmunity can develop slowly to autoimmune-related thyroid dysfunction, perhaps in an early stage only affect- 


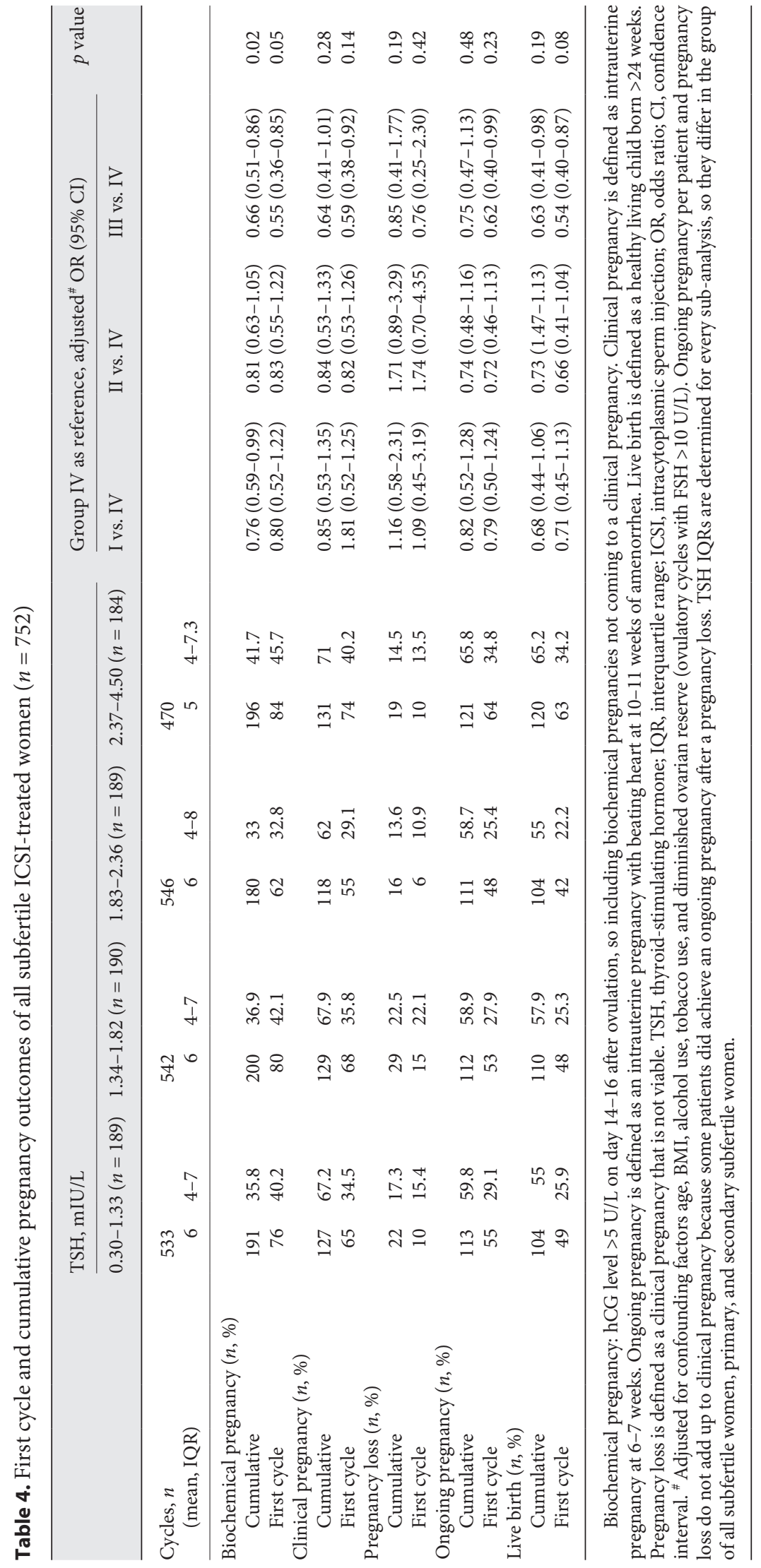




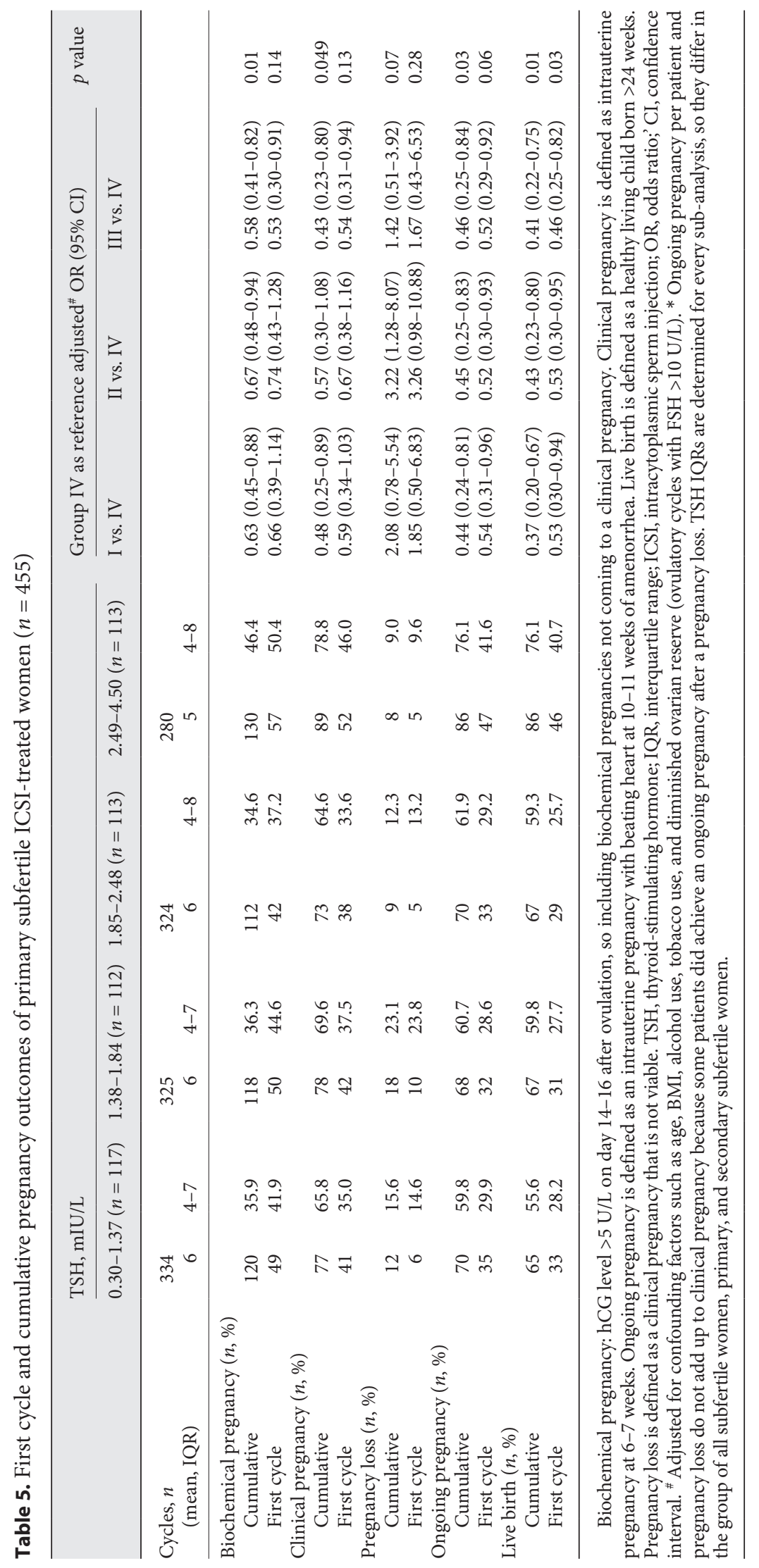




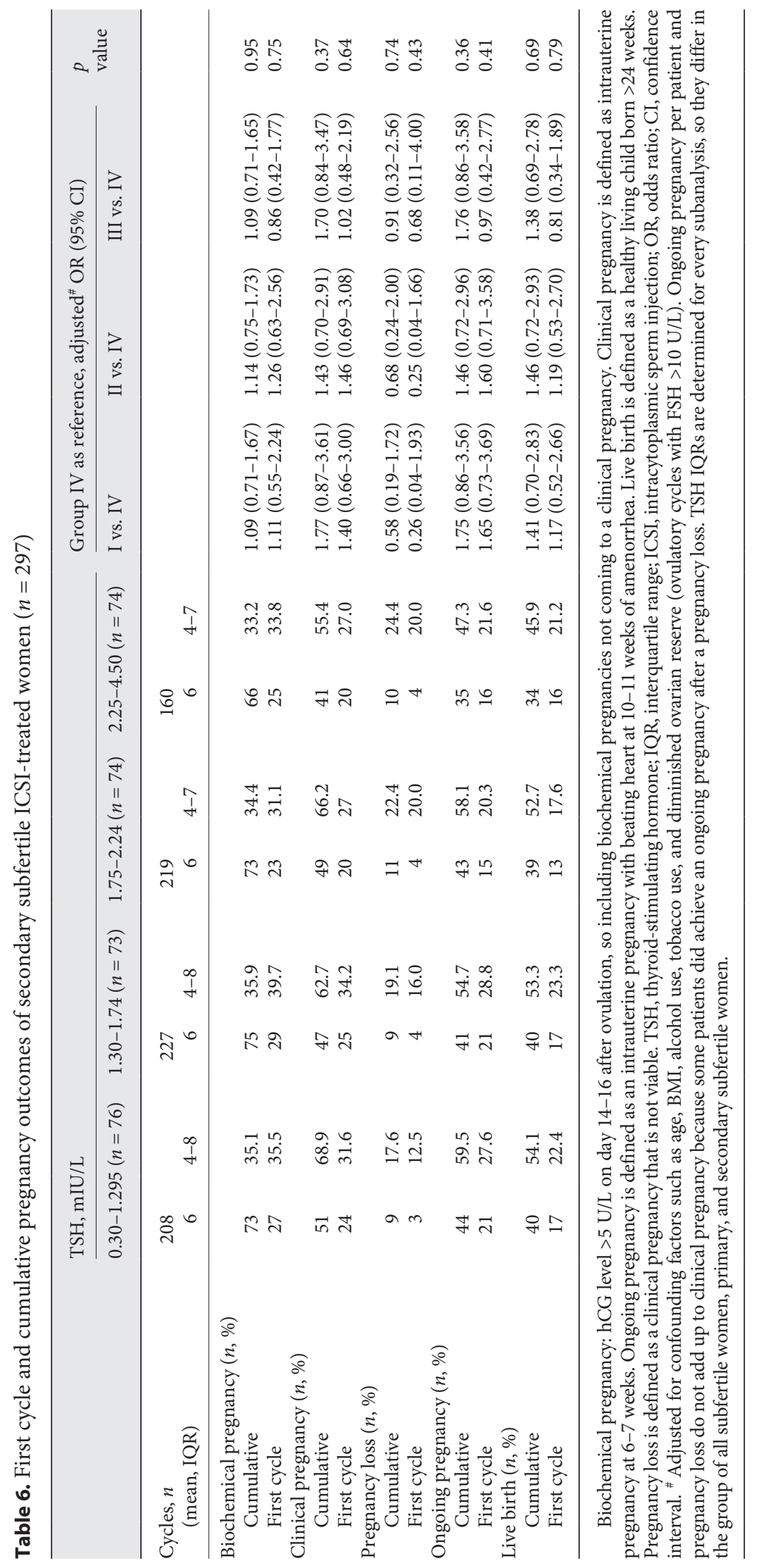


ing fertility [31]. Furthermore, it is known that despite a TSH level in the normal range, thyroid autoantibodies can occupy the zona pellucida [21,32], hampering fertilization by penetration of spermatozoa or be of influence on early embryo development. The latter is in keeping with lesser live births as we found in conventional IVFtreated women with primary unexplained subfertile and upper quartile TSH levels (Repelaer van Driel-Delprat, data submitted). In the previous studies, TPO-Ab positivity was associated with an unfortunate fertility outcome; however, a recent review showed no effect of thyroid autoimmunity on IVF/ICSI [13] and ICSI results [19, 20]. This is in agreement with a possible negative influence of TPO-Ab positivity on the fertility outcome of IVF which can be overcome by ICSI. Of note though, presence of TPO Abs will probably not completely account for our findings since it is known to overall occur in only $10-15 \%$ of the patients.

Unfortunately thyroxine, TPO Abs, and/or thyroglobulin antibodies were not routinely measured in our clinic as we followed NICE and international guidelines $[8,9]$. A strength of our study is the detailed follow-up and the size of the cohort that allowed the subgroup analyses. Moreover, the consistent findings of substantially increased live birth rate in primary subfertile ICSI-treated women with high-normal TSH in different analyses limit the role of chance.

\section{Conclusion}

In this retrospective study, primary subfertile ICSItreated women with higher normal TSH levels have better pregnancy outcomes. These results add to the hypothesis that in certain subfertile women, high but allegedly normal-range TSH levels reflect relative hypothyroidism and/or thyroid autoimmunity, hindering natural fertilization or early embryo development [33]. Though clinical interpretability is still unbalanced, a female factor as described in the ovarian follicle hypothesis by Monteleone [21] and suggested by Poppe [20,33] could benefit from ICSI as treatment for female thyroid dysfunction, hampering conventional (natural or IVF) fertilization, aside from the indication of severe male factor. Therefore, agreeing with the recently published Guideline of the European Thyroid Association [33], we recommend further investigation of the outcome of IVF and ICSI in (primary) subfertile women with high normal TSH levels and/or thyroid autoimmunity, assessed by measuring TPO and thyroglobulin antibodies.

\section{Statement of Ethics}

This study has been formally exempted from ethical approval granted by the Institutional Review Board of the VU University Medical Centre (reference 2013.83, dated March 25, 2013).

\section{Conflict of Interest Statement}

None of the authors had any conflict of interest.

\section{Funding Sources}

The department of Obstetrics and Gynaecology, division of Reproductive Medicine, and of Internal Medicine, division of Endocrinology supported the authors throughout the study period and manuscript preparation.

\section{Author Contributions}

C.R.D.-D. and E.D. designed the study. C.R.D.-D. sought for ethical approval. C.R.D.-D., K.A., M.H., Y.F., A.R., and G.B. collected the data. R.S. supervised the collection of the data. E.D. supervised the research and writing process. P.V. and C.R.D.-D. performed the statistical analysis. C.L. was the supervisor of the study and of critical analysis, interpretation, and presentation of the study.

\section{Data Availability Statement}

The datasets analyzed during the current study are available from the corresponding author on reasonable request.

\section{References}

1 Karmon AE, Cardozo ER, Souter I, Gold J, Petrozza JC, Styer AK. Donor TSH level is associated with clinical pregnancy among oocyte donation cycles. J Assist Reprod Genet. 2016;33(4):489-94.

2 Mandel SJ. Are detection and treatment of thyroid insufficiency in pregnancy feasible? Thyriod. 2005;15(1):44-53.

3 Michalakis KG, Mesen TB, Brayboy LM, Yu B, Richter KS, Levy M, et al. Subclinical elevations of thyroid-stimulating hormone and assisted reproductive technology outcomes. Fertil Steril. 2011;95(8):2634-7.

4 Reh A, Grifo J, Danoff A. What is a normal thyroid-stimulating hormone (TSH) level? Effects of stricter TSH thresholds on pregnancy outcomes after in vitro fertilization. Fertil Steril. 2010;94(7):2920-2.

5 Baloch Z, Carayon P, Conte-Devolx B, Demers LM, Feldt-Rasmussen U, Henry JF, et al. Laboratory medicine practice guidelines. Laboratory support for the diagnosis and monitoring of thyroid disease. Thyroid. 2003; 13(1):3-126. 
6 Dhillon-Smith RK, Tobias A, Smith PP, Middleton LJ, Sunner KK, Baker K, et al. The prevalence of thyroid dysfunction and autoimmunity in women with history of miscarriage or subfertility. J Clin Endocrinol Metab. 2020;105(8):dgaa302.

7 Dhillon-Smith RK, Middleton LJ, Sunner KK, Cheed V, Baker K, Farrell-Carver S, et al. Levothyroxine in women with thyroid peroxidase antibodies before conception. N Engl J Med. 2019;380(14):1316-25.

8 Alexander EK, Pearce EN, Brent GA, Brown RS, Chen H, Dosiou C, et al. 2017 Guidelines of the American Thyroid Association for the Diagnosis and Management of Thyroid Disease During Pregnancy and the Postpartum. Thyroid. 2017;27(3):315-89.

9 Practice Committee of the American Society for Reproductive Medicine. Subclinical hypothyroidism in the infertile female population: a guideline. Fertil Steril. 2015;104(3):545-53.

10 Cramer DW, Sluss PM, Powers RD, McShane P, Ginsburgs ES, Hornstein MD, et al. Serum prolactin and TSH in an in vitro fertilization population: is there a link between fertilization and thyroid function? J Assist Reprod Genet. 2003;20(6):210-5.

11 Andrisani A, Sabbadin C, Marin L, Ragazzi E, Dessole F, Armanini D, et al. The influence of thyroid autoimmunity on embryo quality in women undergoing assisted reproductive technology. Gynecol Endocrinol. 2018;34(9): 752-5.

12 Weghofer A, Himaya E, Kushnir VA, Barad $\mathrm{DH}$, Gleicher N. The impact of thyroid function and thyroid autoimmunity on embryo quality in women with low functional ovarian reserve: a case-control study. Reprod Biol Endocrinol. 2015;13:43.

13 Venables A, Wong W, Way M, Homer HA. Thyroid autoimmunity and IVF/ICSI outcomes in euthyroid women: a systematic review and meta-analysis. Reprod Biol Endocrinol. 2020;18(1):120.

14 van den Boogaard E, Vissenberg R, Land JA, van Wely M, van der Post JA, Goddijn M, et al. Significance of (sub)clinical thyroid dysfunction and thyroid autoimmunity before conception and in early pregnancy: a systematic review. Hum Reprod Update. 2011;17(5): 605-19.
15 Vissenberg R, van den Boogaard E, van Wely $\mathrm{M}$, van der Post JA, Fliers E, Bisschop PH, et al. Treatment of thyroid disorders before conception and in early pregnancy: a systematic review. Hum Reprod Update. 2012;18(4): $360-73$.

16 Maraka S, Singh Ospina NM, Mastorakos G, O'Keeffe DT. Subclinical hypothyroidism in women planning conception and during pregnancy: who should be treated and how? J Endocr Soc. 2018;2(6):533-46.

17 Negro R, Formoso G, Coppola L, Presicce G, Mangieri T, Pezzarossa A, et al. Euthyroid women with autoimmune disease undergoing assisted reproduction technologies: the role of autoimmunity and thyroid function. J Endocrinol Invest. 2007;30(1):3-8.

18 Wang $\mathrm{H}$, Gao H, Chi H, Zeng L, Xiao W, Wang Y, et al. Effect of levothyroxine on miscarriage among women with normal thyroid function and thyroid autoimmunity undergoing in vitro fertilization and embryo transfer: a randomized clinical trial. JAMA. 2017; 318(22):2190-8.

19 Tan S, Dieterle S, Pechlavanis S, Janssen OE, Fuhrer D. Thyroid autoantibodies per se do not impair intracytoplasmic sperm injection outcome in euthyroid healthy women. Eur J Endocrinol. 2014;170(4):495-500.

20 Poppe K, Autin C, Veltri F, Kleynen P, Grabczan L, Rozenberg S, et al. Thyroid autoimmunity and intracytoplasmic sperm injection outcome: a systematic review and meta-analysis. J Clin Endocrinol Metab. 2018. Epub ahead of print.

21 Monteleone P, Parrini D, Faviana P, Carletti E, Casarosa E, Uccelli A, et al. Female infertility related to thyroid autoimmunity: the ovarian follicle hypothesis. Am J Reprod Immunol. 2011;66(2):108-14.

22 Plowden TC, Schisterman EF, Sjaarda LA, Mumford SL. Response to letter: are we really sure that subclinical hypothyroidism and thyroid autoimmunity are not associated with fecundity, pregnancy loss, or live birth? J Clin Endocrinol Metab. 2016;101(6):L87-8.

23 NVOG, richtlijnen voortplantingsgeneeskunde.
24 Vergouw CG, Kieslinger DC, Kostelijk EH, Botros LL, Schats R, Hompes PG, et al. Day 3 embryo selection by metabolomic profiling of culture medium with near-infrared spectroscopy as an adjunct to morphology: a randomized controlled trial. Hum Reprod. 2012; 27(8):2304-11.

25 Korevaar TI, Muetzel R, Medici M, Chaker L, Jaddoe VW, de Rijke YB, et al. Association of maternal thyroid function during early pregnancy with offspring IQ and brain morphology in childhood: a population-based prospective cohort study. Lancet Diabetes Endocrinol. 2016;4(1):35-43.

26 van der Steeg JW, Steures P, Eijkemans MJ, Habbema JD, Hompes PG, Burggraaff JM, et al. Obesity affects spontaneous pregnancy chances in subfertile, ovulatory women. Hum Reprod. 2008;23(2):324-8.

27 Rachdaoui N, Sarkar DK. Effects of alcohol on the endocrine system. Endocrinol Metab Clin North Am. 2013;42(3):593-615.

28 Hornstein MD. Lifestyle and IVF outcomes. Reprod Sci. 2016;23(12):1626-9.

29 Chang Y, Li J, Li X, Liu H, Liang X. Egg quality and pregnancy outcome in young infertile women with diminished ovarian reserve. Med Sci Monit. 2018;24:7279-84.

30 Korevaar TI, Steegers EA, Pop VJ, Broeren MA, Chaker L, de Rijke YB, et al. Thyroid autoimmunity impairs the thyroidal response to human chorionic gonadotropin: two population-based prospective cohort studies. J Clin Endocrinol Metab. 2017;102(1):69-77.

31 Simopoulou M, Sfakianoudis K, Maziotis E, Grigoriadis S, Giannelou P, Rapani A, et al. The impact of autoantibodies on IVF treatment and outcome: a systematic review. Int J Mol Sci. 2019;20(4):892.

32 Zhong Y-P. Relationship between antithyroid antibody and pregnancy outcome following in vitro fertilization and embryo transfer. Int J Med Sci. 2012(9):121-5.

33 Poppe K, Bisschop P, Fugazzola L, Minziori G, Unuane D, Weghofer A. 2021 European Thyroid Association Guideline on Thyroid Disorders prior to and during assisted reproduction. Eur Thyroid J. 2021;9(6):281-95. 